\title{
An Optimal Preventive Maintenance Scheduling Model
}

\author{
XiaoLi Zou \\ School of Civil Engineering and Architecture, Wuhan Polytechnic University, \\ Wuhan 430023, P. R. China \\ xiaolizou@eyou.com
}

Keywords: preventive maintenance; minimum cost; optimal policies

\begin{abstract}
A generalized model for determining minimum-cost preventive maintenance schedules of a maintained reliability system is presented. Through balancing the cost of system failure during operation against the cost of planned maintenance, an optimal policy is established which minimizes the expected cost rate. A simple equation to determine the optimal period between maintenances for a system with the known failure distribution is acquired, and the calculation for the system with Gamma failure distribution is given.
\end{abstract}

\section{Introduction}

Preventive maintenance (PM) of large complex systems such as computers, radars and airplanes is of great importance in reliability theory. PM includes inspection of a system, and repair or replacement of its parts so as to decrease the possibility of system failure during operation. On the other hand, the cost of maintenance increases with the frequency of PM. In order to make the best use of a system, it is necessary to find an optimal inspection strategy by taking into account the cost of the system failure and the cost of planned maintenance.

There are some literatures concerning various PM strategies. PM models with minimal repairs have been studied by Barlow and Hunter [1]. The authors discussed two age-replacement policies: one is useful in maintaining simple systems (with one component) and the other for large and complex systems (with multi-components). Their models have been generalized by Bechelt and Fisher [2] to the case of two types of failure distributions. Park [3] proposed a policy based on the number of minimal repairs performed. Most models are employed to determine optimal PM policy by minimizing the expected total cost during the system life cycle. In this paper, the optimal period between PM is found by minimizing the average cost per unit operation time, i. e. cost rate of the system.

\section{Minimum average cost rate criterion}

Suppose that the failure of a system during operation is immediately identified and $f_{T}(t)$ is the probability density function of the system failure time. Let us call the cost in consequence of failure $C_{F}$ and the cost of PM $C_{M}$, where $C_{F}$ and $C_{M}$ are constants. If the period between the 
present PM and the next is $\tau$, we divide the time interval $[0, \tau]$ into $N$ small time intervals: $\left[0, t_{1}\right], \ldots,\left[t_{i-1}, t_{i}\right], \ldots,\left[t_{N-1}, t_{N}\right]$. The cost per unit operation time of the system, i.e. cost rate is give by

$$
\xi_{\tau}=\sum_{i=1}^{N} \frac{C_{F}}{t_{i}} \mathrm{I}\left\{t_{i-1}<T \leq t_{i}\right\}+\frac{C_{M}}{\tau} \mathrm{I}\{T>\tau\}
$$

where $\mathrm{I}\{A\}$ is an indicator function of event $A$ and $T$ the system failure time..

The Eq. 1 means that if failure has occurred at time interval $\left[t_{i-1}, t_{i}\right]$, then the cost rate becomes equal to $C_{F} / t_{i}$, and if after period $\tau$ when PM is executed, the cost rate will be equal to $C_{M} / \tau$. The cost rate $\xi_{\tau}$ is a random variable and its expected value, i.e. average cost rate is

$$
E \xi_{\tau}=\sum_{i=1}^{N} \frac{C_{F}}{t_{i}} \mathrm{P}\left\{t_{i-1}<T \leq t_{i}\right\}+\frac{C_{M}}{\tau} \mathrm{P}\{T>\tau\}
$$

The small time interval length $\Delta t=t_{i}-t_{i-1}$ decreases with the increase of the number $N$. We have

$$
\mathrm{P}\left\{t_{i-1}<T \leq t_{i}\right\} \approx f_{T}(t) \Delta t
$$

So when $N \rightarrow \infty$, Eq. 2 becomes

$$
E \xi_{\tau}=\int_{0}^{\tau} \frac{C_{F}}{t} f_{T}(t) d t+\frac{C_{M}}{\tau} \int_{\tau}^{\infty} f_{T}(t) d t
$$

The optimal period $\tau^{*}$ can be obtained by setting the partial derivative of $E \xi_{\tau}$ with respect to $\tau$ zero and solving the equation, i.e.

$$
\frac{d E \xi_{\tau}}{d \tau}=\frac{C_{F}-C_{M}}{\tau} f_{T}(\tau)-\frac{C_{M}}{\tau^{2}} \int_{\tau}^{\infty} f_{T}(t) d t=0
$$

\section{Numerical example}

Assume a two parameter Gamma distribution for the system failure. Gamma distribution is a natural extension of the exponential distribution. If the shocks to a system occur independently at the same rate $\lambda$ and the system will occur failure after $\alpha$ shocks, then the system failure time $T$ is the Gamma distribution. The probability density function is

$$
f_{T}(t)=\frac{\lambda^{\alpha} t^{\alpha-1}}{\Gamma(\alpha)} e^{-\lambda t} \quad t>0
$$

Where $\lambda$ is the scale parameter; $\alpha$ the shape parameter, and $\Gamma(\alpha)$ the Gamma function, i.e.

$$
\Gamma(\alpha)=\int_{0}^{\infty} x^{\alpha-1} e^{-x} d x
$$

Assume $\alpha=2$, Eq. 6 becomes

$$
f_{T}(t)=\frac{\lambda^{2} t}{\Gamma(2)} e^{-\lambda t}=\lambda^{2} t e^{-\lambda t}
$$

Substituting $f_{T}(t)$ into Eq. 5 yields 


$$
\left(C_{F}-C_{M}\right) \lambda^{2} e^{-\lambda \tau}-\frac{C_{M}}{\tau} \int_{\tau}^{\infty} \lambda^{2} t e^{-\lambda t} d t=0
$$

where $\int_{\tau}^{\infty} \lambda^{2} t e^{-\lambda t} d t=\lambda \tau e^{-\lambda \tau}-e^{-\lambda \tau}$. Eq. 9 can be rewritten as

$$
\left(\frac{C_{F}}{C_{M}}-1\right) \lambda \tau^{2}-\lambda \tau-1=0
$$

So the optimal period between PM is

$$
\tau^{*}=\frac{C_{M}+\sqrt{C_{M}\left(4 C_{F}-3 C_{M}\right)}}{2 \lambda\left(C_{F}-C_{M}\right)}
$$

Substituting $\tau^{*}$ into Eq. 4, it gives the corresponding average cost rate $E \xi_{\tau^{*}}$. The results of

\begin{tabular}{|c|c|c|c|c|c|c|c|c|c|c|}
\hline \multirow{2}{*}{$C_{F}$} & \multicolumn{2}{|c|}{$\lambda=0.1$} & \multicolumn{2}{|c|}{$\lambda=0.2$} & \multicolumn{2}{|c|}{$\lambda=0.3$} & \multicolumn{2}{|c|}{$\lambda=0.4$} & \multicolumn{2}{|c|}{$\lambda=0.5$} \\
\hline & $\tau^{*}$ & $E \xi_{\tau^{*}}$ & $\tau^{*}$ & $E \xi_{\tau^{*}}$ & $\tau^{*}$ & $E \xi_{\tau^{*}}$ & $\tau^{*}$ & $E \xi_{\tau^{*}}$ & $\tau^{*}$ & $E \xi_{\tau^{*}}$ \\
\hline 20 & 16.18 & 1.92 & 8.09 & 3.85 & 5.39 & 5.77 & 4.05 & 7.70 & 3.24 & 9.62 \\
\hline 30 & 10.00 & 2.63 & 5.00 & 5.26 & 3.33 & 7.90 & 2.50 & 10.53 & 2.00 & 13.16 \\
\hline 40 & 7.68 & 3.21 & 3.84 & 6.42 & 2.56 & 9.64 & 1.92 & 12.85 & 1.54 & 16.06 \\
\hline 50 & 6.40 & 3.71 & 3.20 & 7.43 & 2.13 & 11.14 & 1.60 & 14.86 & 1.28 & 18.57 \\
\hline 60 & 5.58 & 4.16 & 2.79 & 8.33 & 1.86 & 12.49 & 1.40 & 16.66 & 1.12 & 20.82 \\
\hline 70 & 5.00 & 4.57 & 2.50 & 9.15 & 1.67 & 13.72 & 1.25 & 18.30 & 1.00 & 22.07 \\
\hline 80 & 4.56 & 4.95 & 2.20 & 9.91 & 1.52 & 14.86 & 1.14 & 19.81 & 0.91 & 24.77 \\
\hline 90 & 4.22 & 5.31 & 2.11 & 10.62 & 1.41 & 15.92 & 1.05 & 21.23 & 0.84 & 26.54 \\
\hline 100 & 3.93 & 5.64 & 1.97 & 11.28 & 1.31 & 16.93 & 0.98 & 22.57 & 0.79 & 28.21 \\
\hline 110 & 3.70 & 5.96 & 1.85 & 11.92 & 1.23 & 17.88 & 0.93 & 23.84 & 0.74 & 29.80 \\
\hline
\end{tabular}
calculations are shown in Table 1.

Table 1 Optimal period between PM and average cost rate for ( $\alpha=2 ; C_{M}=10$ )

The following interpretations can be given to the data in Table 1.

(1) By holding the cost of PM $\left(C_{M}\right)$ constant, a system with high cost of failure $\left(C_{F}\right)$ will require frequent maintenance. This will increase the average cost rate of operating the system. For example, for a system with $\lambda=0.1$, it requires PM of 16.18 time units during the system operation at the average cost rate of 1.92, given $C_{F}=20$. However, for the case where $C_{F}=100$, PM of 3.93 time units at the average cost rate of 5.64 is incurred.

(2) The period between PM $\left(\tau^{*}\right)$ decreases for a system with decreasing value of $\lambda$. That is, the smaller the amount of failure a system generates during its operation, the less frequent is the maintenance it may need. For example, for $\lambda=0.1$ (i.e. one failure per 10 time units), $\tau^{*}$ has 
a value of 5.58; $E \xi_{\tau^{*}}=4.16$, and for $\lambda=0.5$ (i.e. five failures per 10 time units), $\tau^{*}$ has a value of $1.12 ; E \xi_{\tau^{*}}=20.82$.

\section{Conclusions}

This paper has established a minimum average cost rate criterion for determining economic PM schedules of maintained reliability systems. Compared with existing models, the minimum average cost rate criterion is natural and the equation for calculating the optimal PM period is simple. The application of the approach to a system with Gamma type failure distribution is presented. Gamma distribution is selected over other distributions because of its versatility in describing cumulative system shocks. The computational results are shown that a system with higher failure rate and cost would require more frequent maintenance.

\section{References}

[1] R.E. Barlow, L.C. Hunter, Optimum preventive maintenance policies [J]. Operations Research, 1960 (9) 90-100.

[2] F. Beichelt, K.G. Fisher, General failure model applied to preventive maintenance [J]. IEEE Trans. Reliability, 1980 (29) 39-41.

[3] K.S. Park, Optimum number of minimal repairs before replacement [J]. IEEE Trans. Reliability, 1979 (28) 137-140.

[4] V.A. Kopnov, Residual life, linear fatigue damage accumulation and optimal stopping [J]. Reliability Engineering and System Safety, 1993 (40) 319-325.

[5] T. Nakagawa, Periodic and sequential preventive maintenance policies [J]. J. Appl. Prob. 1986 (23) 536-542. 